\title{
Frenectomia para restituição fono-motricial da língua
}

\author{
Phrenectomy for phonetic and motor tongue restoration
}

Frenectomía para la restauración fonética y motora de la lengua

\begin{abstract}
Ana Lídia Soares Cota ${ }^{1 *}$, Ávila Vanderlei de Lima ${ }^{1}$, Stephanie Karolyne dos Santos Pereira ${ }^{1}$, Mariana Alencar Nemezio".
\end{abstract}

\section{RESUMO}

Objetivo: Relatar o caso clínico de frenectomia lingual em uma criança com anquiloglossia, visando sua restituição fono-motricial. Descrição do caso: Paciente do sexo masculino, melanorderma, sete anos de idade, com queixa de bullying no ambiente escolar devido à dificuldade na dicção. Ao exame clínico, observou-se o freio lingual curto e fixado ao ápice da língua, resultando na limitação da protrusão e da projeção da língua em direção ao palato duro, além do comprometimento na dicção de alguns fonemas, confirmando o diagnóstico de anquiloglossia. Como conduta terapêutica, optou-se pela intervenção cirúrgica convencional (frenectomia). No pós-operatório imediato foi observado o freio centralizado no ventre lingual, bem como a eficiência na elevação e protrusão da língua. Uma vez que o paciente continuava com comprometimento fonético, o mesmo foi encaminhado para fonoterapia. Durante o acompanhamento pôdese constatar melhora na fonação e ausência de queixas relacionadas aos aspectos comportamentais e convívio escolar. Considerações finais: A frenectomia de um freio lingual curto, tardiamente diagnosticado, apresenta-se como uma técnica cirúrgica de fácil execução e com potencial para restabelecer as funções motriciais da língua. Além disso, a instituição de uma abordagem multiprofisional, associando intervenção cirúrgica à fonoaudiológica, contribuiu para a restituição fonética e reinserção social do paciente.

Palavras-chave: Anquiloglossia, Procedimentos Cirúrgicos Bucais, Freio Lingual.

\begin{abstract}
Objective: To report the clinical case of lingual frenectomy in a child with ankyloglossia, aiming at its phonetic and motor restoration. Case description: Male melanorderma patient, seven years old, complaining of bullying in the school environment due to difficulty in diction. On clinical examination, a short lingual brake fixed to the apex of the tongue was observed, resulting in limitation of protrusion and elevation of the tongue towards the hard palate, in addition to the impairment of some phonemes, confirming the diagnosis of ankyloglossia. As a therapeutic approach, we opted for the conventional surgical intervention (frenectomy). In the immediate postoperative, the centralized brake on the lingual belly was observed, as well as the efficiency in the elevation and protrusion of the tongue. Once the patient continued with phonetic impairment, he was referred to speech therapy. During follow-up, it was observed improvement in phonation and absence of complaints related to behavioral aspects and school life. Final considerations: The late diagnosed frenectomy of a short lingual brake is an easily performed surgical technique to reestablish the tongue's motor functions. In addition, the establishment of a multiprofessional approach, associating surgical intervention with speech therapy, contributed to the phonetic restitution and social reintegration of the patient.
\end{abstract}

Key words: Ankyloglossia, Oral Surgical Procedures, Lingual Brake.

${ }^{1}$ Centro Universitário Tiradentes (UNIT/AL), Maceió-Alagoas. *E-mail: ana.cota@uol.com.br

SUBMETIDO EM: 8/2019

ACEITO EM: 9/2019

PUBLICADO EM: 10/2019

REAS/EJCH | Vol.Sup.35 | e1457 | DOI: https://doi.org/10.25248/reas.e1457.2019 Página 1 de 7 


\section{RESUMEN}

Objetivo: Describir el caso clínico de frenectomía lingual en un niño con anquiloglosia. Descripción del caso: Paciente masculino de siete años, quejándose de acoso escolar debido a la dificultad en la dicción. En el examen clínico, se observó un frenillo lingual corto fijado al vértice de la lengua, lo que resultó en la limitación de la protrusión y la protrusión de la lengua hacia el paladar duro, además del deterioro de algunos fonemas, lo que confirma el diagnóstico de anquiloglosia. Como enfoque terapéutico, optamos por la intervención quirúrgica convencional (frenectomía). En el postoperatorio inmediato, se observó el frenillo centralizado en el vientre lingual, así como la eficiencia en la elevación y protrusión de la lengua. El paciente continuó con discapacidad fonética y fue derivado a terapia del habla. Durante el seguimiento, se observó una mejora en la fonación y la ausencia de quejas relacionadas con los aspectos conductuales y la vida escolar. Consideraciones finales: La frenectomía diagnosticada tardía de un frenillo lingual corto es una técnica quirúrgica con el potencial de restablecer las funciones motoras de la lengua. Además, el establecimiento de un enfoque multiprofesional, que asocia la intervención quirúrgica y terapia del habla, contribuyó a la restitución fonética y la reintegración social del paciente.

Palabras clave: Anquiloglosia, Procedimientos Quirúrgicos Orales, Frenillo Lingual.

\section{INTRODUÇÃO}

O freio ou frênulo lingual é uma prega conjuntiva que se insere a partir do ventre da língua, entre o ápice e o terço médio, até o assoalho da boca, permitindo a livre movimentação de sua porção anterior (BRAGA LAS, et al., 2009).

Em geral, uma modificação dessa estrutura pode ser detectada quando uma pequena porção de tecido embrionário, que deveria ter sofrido apoptose durante o desenvolvimento, continua na sua face ventral (GOMES E, et al., 2015; POMPÉIA LE, et al., 2017).

A anquiloglossia, popularmente conhecida como "língua presa", refere-se a uma alteração congênita no freio lingual associada a sua fixação, caso seja anteriorizada e muito próxima ao ápice da língua, e/ou a sua extensão, relativa ao tamanho reduzido, o que pode gerar diferentes graus de limitações dos movimentos linguais (BRAGA LAS, et al., 2009; VIEIRA EM et al., 2010; MELO NSFO, et al., 2011; GOMES E, et al., 2015; SUZART DD e CARVALHO ARR, 2016).

O fator etiológico específico da anquiloglossia é desconhecido, mas sugere-se a influência de um gene autossômico dominante herdado geneticamente (VIEIRA AR et al., 2014; SILVA PI et al., 2016). Ademais, o consumo materno de cocaína durante a gravidez é relatado como um fator de risco, aumentando três vezes a chance de o recém-nascido apresentar alteração no freio lingual (SANTOS POM, et al., 2018).

Estudos epidemiológicos apontam uma prevalência de 0.1 a $10.7 \%$ na população, sendo esta variação relacionada às controvérsias acerca do diagnóstico, uma vez que são utilizados critérios subjetivos, tornando este processo dificilmente uniforme (LEAL RAS, 2010; RINALDI G, 2013; XAVIER MMAPC, 2014; GOMES E, et al., 2015).

A opção terapêutica mais indicada para correção da anquiloglossia é a remoção cirúrgica do freio lingual, denominada frenectomia (SILVA PI, et al., 2016). Entretanto, em alguns casos, a condição é tratada em associação ou somente com intervenções mais conservadoras, como a fonoterapia, visando à melhora das funções estomatognáticas, dentre elas a correção da fonação, mastigação e postura anormal da língua (VIEIRA AR, et al., 2014; SUZART DD e CARVALHO ARR, 2016; OLIVEIRA DV, et al., 2019).

Além do comprometimento funcional da língua, a referida condição apresenta potencial para causar importante prejuízo social, tendo em vista que muitas crianças podem ser vítimas de bullying devido à dificuldade na dicção de algumas palavras (SILVA PI, et al., 2016; SANTOS POM, et al., 2018).

Diante do exposto, o presente trabalho tem como objetivo relatar um caso clínico de frenectomia lingual em uma criança com anquiloglossia, visando sua restituição fono-motricial. 


\section{DETALHAMENTO DO CASO}

Paciente do sexo masculino, melanorderma, sete anos de idade, compareceu à Clínica de Odontopediatria do Centro Universitário Tiradentes (UNIT/AL) com queixa principal de bullying no ambiente escolar devido à dificuldade na dicção de algumas palavras.

Todo o atendimento odontológico foi precedido da autorização do responsável legal pelo paciente, mediante assinatura do termo de consentimento livre e esclarecido.

Durante a anamnese, constatou-se que o menor possuía boa saúde geral, ausência de doenças sistêmicas ou alergias e não fazia uso de nenhum medicamento. Ao exame clínico, observou-se o freio lingual curto e fixado ao ápice da língua, resultando na limitação da projeção da língua em direção ao palato duro (Figura 1).

Figura 1 - Limitação da projeção da língua em direção ao palato duro em decorrência do freio curto e fixado ao ápice lingual.

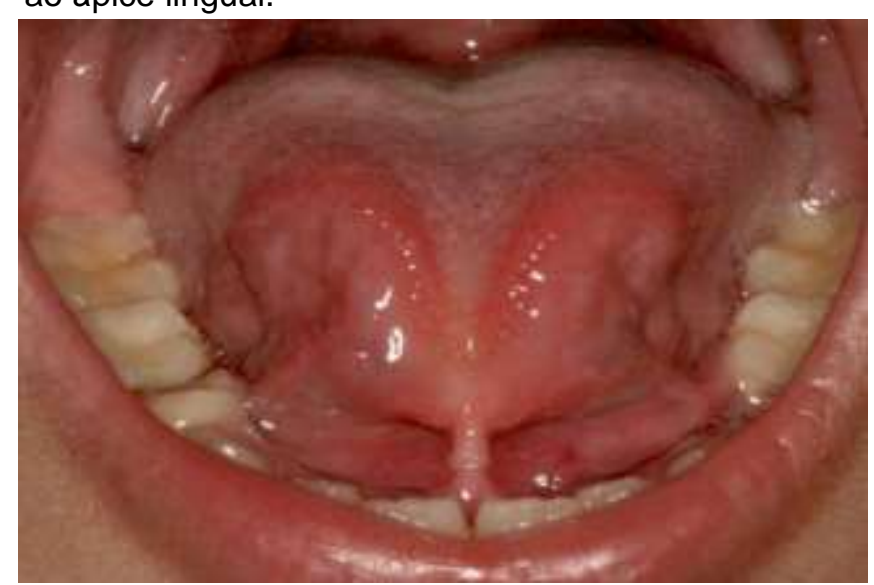

Fonte: Cota ALS, Lima AV, Pereira SKS, et al., 2019.

No decorrer do movimento de protrusão lingual, notou-se a formação de uma dobra, conferindo um formato de coração em seu ápice, contribuindo assim para a confirmação do diagnóstico de anquiloglossia (Figura 2). Além das restrições da motricidade lingual, também foi detectado um comprometimento na dicção dos fonemas com som de /s/ e /r/.

Figura 2 - Limitação da protrusão da língua conferindo um formato de coração.

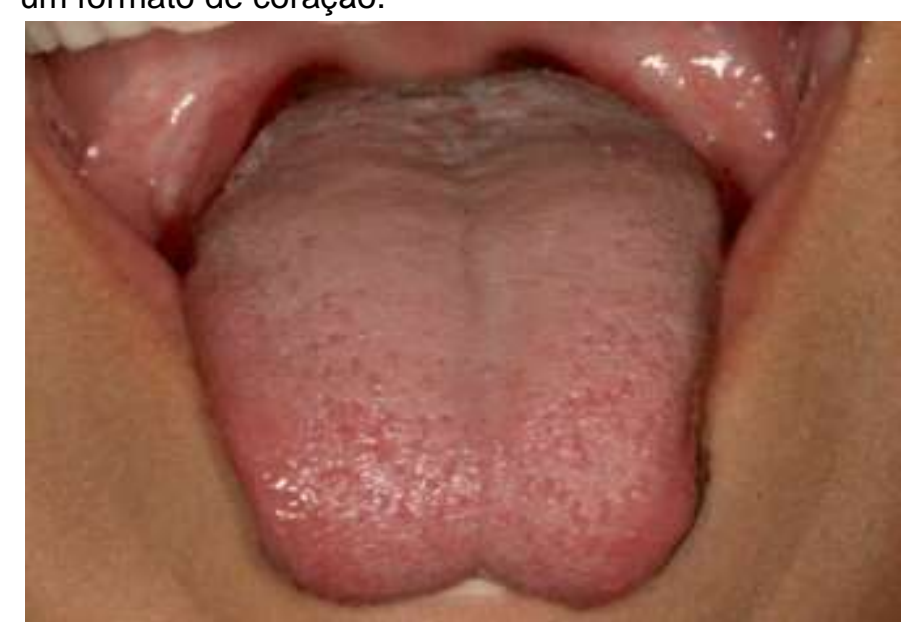

Fonte: Cota ALS, Lima AV, Pereira SKS, et al., 2019. 
Como conduta terapêutica, optou-se pela intervenção cirúrgica via técnica convencional, caracterizada pela remoção total do freio lingual (frenectomia). Inicialmente foi realizada antissepsia extra e intraoral com digluconato de clorexidina 2\%, seguida de anestesia local com Mepivacaína a 2\% com Epinefrina 1:100.00 pela técnica infiltrativa dos nervos linguais, em ambos os lados do freio. Durante esta manobra, os operadores tiveram cuidado para não deformar o freio lingual, o que poderia dificultar a visualização do ponto de referência para sua incisão. A imobilização da língua, visando seu tracionamento para melhor visualização do campo cirúrgico, foi obtida com auxílio de um fio de sutura transfixado no ápice lingual. Em seguida, realizou-se uma pequena incisão na porção mediana do freio lingual com tesoura cirúrgica reta e divulsão das fibras musculares com tesoura de ponta romba (Figura 3). Por fim, foi conduzida a sutura da ferida cirúrgica pela técnica de ponto simples, com fio de seda.

Figura 3 - Incisão com tesoura de ponta reta na região mediana do freio lingual.

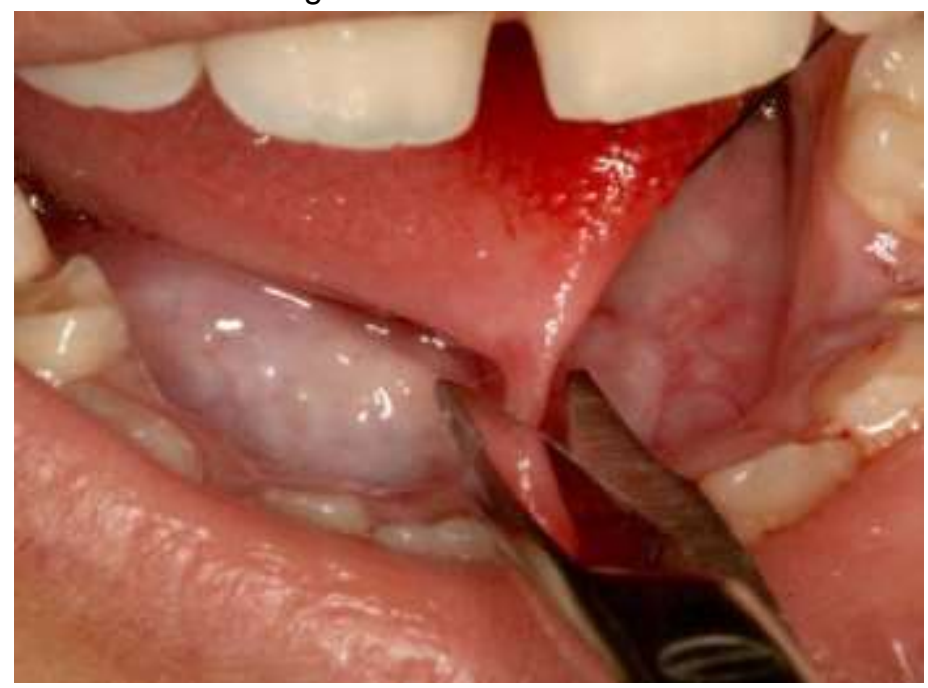

Fonte: Cota ALS, Lima AV, Pereira SKS, et al., 2019.

No pós-operatório imediato foi observado o freio centralizado no ventre lingual e não mais no ápice, bem como a eficiência na elevação e protrusão da língua (Figuras 4 e 5). As orientações pós-cirúrgicas incluíram dieta líquida e/ou pastosa, alimentos frios e repouso físico. Como o paciente continuava com comprometimento fonético, o mesmo foi encaminhado para acompanhamento fonoudiológico.

Figura 4 - Pós-operatório imediato evidenciando a projeção da língua em direção ao palato duro.

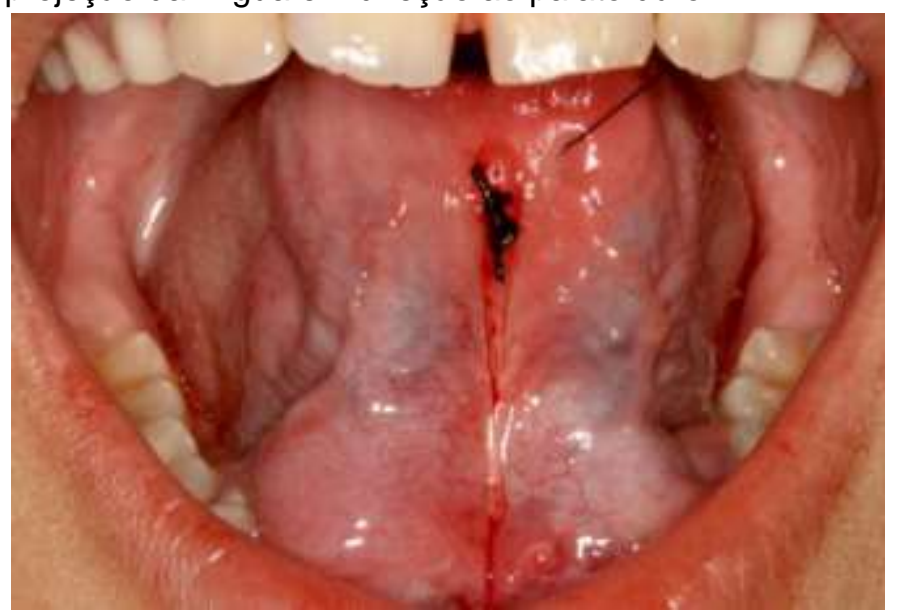

Fonte: Cota ALS, Lima AV, Pereira SKS, et al., 2019. 
Figura 5 - Pós-operatório imediato evidenciando a protrusão da língua, sem tracionamento ocasionado pelo freio curto.

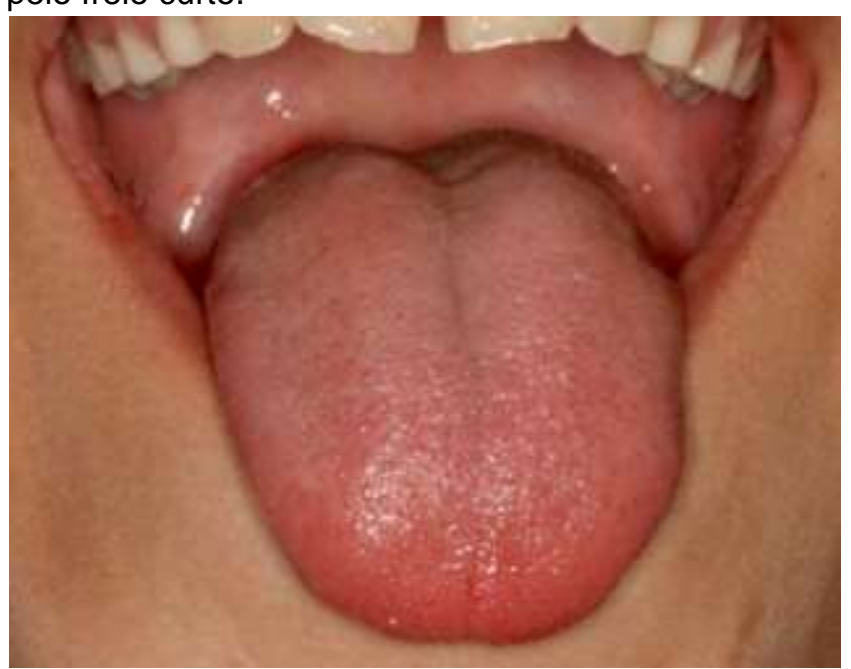

Fonte: Cota ALS, Lima AV, Pereira SKS, et al., 2019.

O paciente foi acompanhado clinicamente por até 6 meses, quando pôde-se constatar uma melhora na fonação e ausência de queixas relacionadas aos aspectos comportamentais e convívio escolar (Figura 6).

Figura 6 - Aspecto cicatricial após 6 meses de acompanhamento com destaque para a inserção centralizada do freio no ventre da língua.

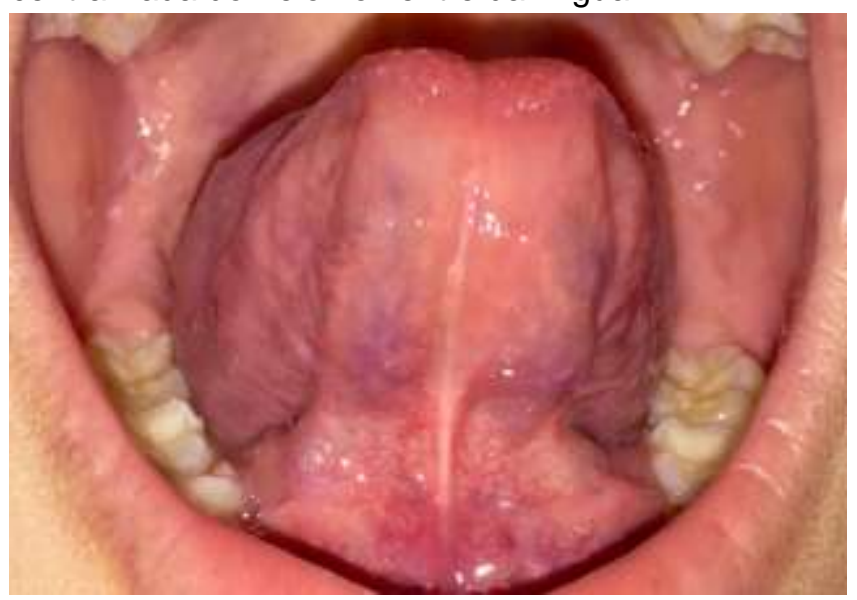

Fonte: Cota ALS, Lima AV, Pereira SKS, et al., 2019.

\section{DISCUSSÃO}

O presente caso clínico reporta-se a um paciente do gênero masculino, corroborando a literatura a qual aponta que os homens são mais afetados que as mulheres, em uma proporção de 1:3 (BRAGA LAS, et al., 2009; LEAL RAS, 2010; GOMES E, et al., 2015; SANTOS POM, et al., 2018). O diagnóstico de anquiloglossia tem sido realizado após exame clínico acurado, com avaliação do comprimento e fixação do freio e mobilidade lingual (SILVA PI et al., 2016).

Sua identificação precoce é padrão-ouro para estabelecimento de uma adequada terapêutica e, deve ocorrer, preferencialmente, em pacientes neonatos para prevenir intercorrências durante a amamentação, como trauma mamilar maternal e baixo peso infantil (VIEIRA AR et al., 2014; SILVA PI et al., 2016; OLIVEIRA DV et al., 2019). 
Neste sentido, no dia 20 de junho de 2014 foi sancionada a Lei Federal ํㅡ⒔002, tornando-se obrigatória a avaliação do freio lingual em todos os bebês nascidos em maternidades, hospitais ou outras dependências do território brasileiro. O denominado "Teste da Linguinha" é um exame simples e indolor que deve ser realizado nas primeiras 48 horas após o nascimento do bebê. Por meio de uma avaliação anatomofuncional da língua, pode ser indicada a realização de intervenção cirúrgica ainda no local de nascimento (MARTINELLI RLC et al., 2012; MARTINELLI RLC et al., 2016).

Vargas BC, et al. (2008) reforçam que, com base no modelo assistencial de saúde voltado para o diagnóstico precoce e pronto tratamento das alterações buco-dentárias, o acompanhamento pré-natal odontológico e as consultas neonatais com um odontopediatra deveriam ser inseridas na rotina de cuidados da puericultura propiciando que os pais sejam comunicados acerca da presença de anormalidades e seus problemas potenciais.

Não existe um consenso sobre a idade ideal para a intervenção cirúrgica de um freio lingual curto. Entretanto, a frenectomia é indicada sempre que houver prejuízo funcional ao indivíduo (LEAL RAS, 2010; SANTOS POM, et al., 2018). Recomenda-se que em crianças após a fase de amamentação e com dúvida no diagnóstico, a indicação da cirurgia seja postergada e instituída uma abordagem conservadora com foco no acompanhamento clínico, visto que os problemas de fala e mecânicos/sociais podem ou não se desenvolver ao longo dos anos (VARGAS BC, et al., 2008).

No caso relatado, a detecção tardia da anquiloglossia implicou o comprometimento motricial da língua e, consequentemente, da dicção. $O$ freio curto e fixado no ápice lingual desencadeou dificuldade na pronúncia e articulação de palavras labiodentais, repercutindo na socialização e autoestima do paciente. Assim sendo, a frenectomia foi adotada como conduta terapêutica, permitindo o reestabelecimento imediato da mobilidade lingual. Cabe ressaltar que tal condição também pode gerar outras consequências relevantes e de impactos físicos e sociais, como desconforto local, diastema entre dentes incisivos inferiores, impedimentos na manutenção da higiene oral, impossibilidade de realizar tarefas simples, como tocar instrumentos de sopro, e problemas de comportamento que podem potencializar a geração de constrangimento social durante a infância e adolescência, devido ao desencadeamento de episódios de bullying (VIERA JIMP, 2012; RINALDI G, 2013).

Após o procedimento, o paciente foi submetido a testes para averiguar a motricidade da língua, de forma que o mesmo conseguiu realizar com eficiência os movimentos de protrusão, lateralidade e elevação lingual, antes impedidos pelo freio encurtado. Cabe salientar que, além da técnica convencional descrita, o laser cirúrgico, como o Nd:YAP, vem sendo empregado como recurso terapêutico em frenectomias, apresentando excelentes condições trans e pós-operatórias. Sua transmissão por fibra óptica de sílica promove uma incisão apurada e resulta em ausência ou mínima ocorrência de sangramento, edema e dor pós-operatória (LOKKI DTL, 1996; SANTOS ESR, et al., 2007).

Neste trabalho, a restituição fonética não ocorreu de forma imediata, ou seja, mesmo após a correção da fixação do freio lingual o paciente permaneceu com deficiência na diç̧ão dos fonemas $/ \mathrm{s} / \mathrm{e} / \mathrm{r} /$. Portanto, uma terapia complementar foi indicada para aprimorar a reprodução fonética através de acompanhamento com fonoaudiólogo. Sabe-se que esta estratégia beneficia o paciente adaptando-o às modificações provocadas pela intervenção cirúrgica (BRITO SF et al., 2008; GOMES E, et al., 2015).

Desta forma, considerando que algumas crianças diagnosticadas com anquiloglossia podem ter a fala normal apesar da mobilidade restrita da língua, torna-se prudente a integralidade entre os profissionais de diferentes áreas da saúde para a elaboração de uma correta conduta clínica, viabilizando sempre o bem-estar do paciente (IZOLANI NETO O, et al., 2014).

Pode-se concluir que a frenectomia de um freio lingual curto, tardiamente diagnosticado, apresenta-se como uma técnica cirúrgica de fácil execução e com potencial para restabelecer as funções motriciais da língua. Além disso, a instituição de uma abordagem multiprofissional, associando intervenção cirúrgica à fonoaudiológica, contribuiu para a restituição fonética e reinserção social do paciente. 


\section{REFERÊNCIAS}

1. BRAGA LAS, et al. Prevalência de alteração no frênulo lingual e suas implicações na fala de escolares. Revista CEFAC, 2009; 11(3): 378-390.

2. BRITO SF, et al. Frênulo lingual: classificação e conduta segundo ótica fonoaudiológica, odontológica e otorrinolaringológica. Revista CEFAC, 2008; 10(3): 343-351.

3. GOMES E, et al. Freio lingual: abordagem clínica interdisciplinar da fonoaudiologia e odontopediatria. Revista da Associação Paulista de Cirurgiões Dentistas, 2015; 69(1): 20-24.

4. LEAL RAS. Frenectomia lingual e labial em odontopediatria. Porto. Monografia de Investigação/Relatório de Actividade Clínica. Faculdade de Medicina Dentária - Universidade do Porto; 2010.

5. LOKKI DTL. Laser para clínica geral em odontologia. Manual do proprietário. 1996.

6. MARTINELLI RLC, et al. Protocolo de avaliação do frênulo da língua em bebês. Revista CEFAC, 2012; 14(1): 138145.

7. MARTINELLI RLC, et al. Validade e confiabilidade da triagem: "teste da linguinha". Revista CEFAC, 2016; 18(6): 1323-1331.

8. MELO NSFO, et al. Anquiloglossia: relato de caso. Revista Sul-Brasileira de Odontologia, 2011; 8(1): $102-107$.

9. OLIVEIRA DV, et al. Anquiloglossia, tratamento cirúrgico: Relato de caso clínico. Revista de Ciências da Saúde da Amazônia, 2019; 1:76-82.

10. ORLANDO IN, et al. Frenectomia: revisão de literatura. Uningá Review, 2014; 18(3): 21-25.

11. POMPÉIA LE, et al. A influência da anquiloglossia no crescimento e desenvolvimento do sistema estomatognático. Revista Paulista de Pediatria, 2017; 35(2): 216-221.

12. RINALDI G. Modificações anatômicas e funcionais após frenectomia lingual: relato de casos clínicos. Monografia (Especialização em Odontopediatria). Universidade Federal do Rio Grande do Sul, Porto Alegre. 2013; 27 p.

13. SANTOS ESR, et al. Frenectomia a laser (Nd: YAP) em odontopediatria. Revista Odonto, 2007; 15(29): $107-113$.

14. SANTOS POM, et al. Frenulotomia lingual em paciente pediátrico: relato de caso. Archives of Health Investigation, 2018; 7(4): 139-142.

15. SILVA PI, et al. Frenectomia lingual em bebê: relato de caso. Revista Bahiana de Odontologia, 2016; 7(3): $220-227$.

16. SUZART DD, CARVALHO ARR. Alterações de fala relacionadas às alterações do frênulo lingual em escolares. Revista CEFAC, 2016; 18(6): 1332-1339.

17. VARGAS BC, et al. Anquiloglossia: quando indicar a frenectomia lingual? Uningá Review, 2008; 18: 161-170.

18. VIEIRA AR, et al. A importância do tratamento da anquiloglossia e sua relação com o comportamento sexual futuro. Revista Gaúcha de Odontologia, 2014; 52(2): 72-73.

19. VIEIRA EM, et al. Frequência de anquiloglossia em uma comunidade indígena brasileira. Revista Gaúcha de Odontologia, 2010; 58(2): 215-218.

20. VIEIRA JIMP. Tecnologia Laser em Medicina Dentária-Frenectomia em Foco. Universidade Fernando Pessoa, Faculdade de Ciências da Saúde, Porto, 2012.

21. XAVIER MMAPC. Anquiloglossia em pacientes pediátricos. Lisboa. Dissertação (Mestrado Integrado em Medicina Dentária). Universidade de Lisboa, 2014. 